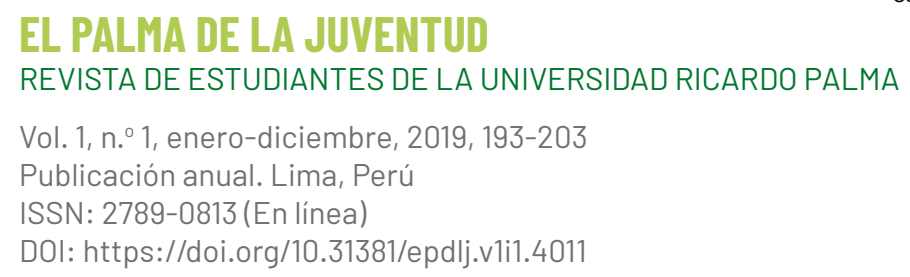

\title{
EL LIDERAZGO DURANTE LA COLONIA EN LAS TRADICIONES PERUANAS DE RICARDO PALMA'
}

\section{The leadership during the Colony in the Tradiciones peruanas by Ricardo Palma}

ROBERTO AGUSTÍN LARA VALLADOLID

Facultad de Ingeniería, Universidad Ricardo Palma

Lima, Perú

Contacto: roberto.lara@urp.edu.pe

\section{RESUMEN}

En este artículo se analizarán las tradiciones «Un virrey hereje y un campanero bellaco» $\mathrm{y}$ «La desolación de Castrovirreina». El tradicionista propone en estos textos demostrar cómo ejercían el liderazgo los virreyes durante la colonia.

Palabras clave: Tradiciones peruanas; liderazgo; virrey.

1 Este artículo se elaboró como parte del curso Taller de Comunicación Oral y Escrita I, asignatura dictada por la profesora Vilma Vera Collazos Alarcón en el semestre 2019-II. 


\section{ABSTRACT}

This article will analyze the traditions «Un virrey hereje y un campanero bellaco» and «La desolación de Castrovirreina». In these texts, the traditionalist proposes to demonstrate how viceroys exercised leadership during the Colony.

Key words: Tradiciones peruanas; leadership; viceroy.

\section{Recibido: 8/11/2019 Aceptado: 8/12/2019}

\section{INTRODUCCIÓN}

Durante la etapa de evolución tecnológica que ha vivido el hombre, este ha avanzado dentro de sus diferentes entornos, de tal manera que ha generado ciertas cualidades que le permiten destacar dentro de un grupo y ser innovador con respecto a planificar métodos y diversas acciones dentro de un sector, además de crear objetivos y metas claras para lograr capacidades en sus seguidores. Aquellas características son propias de personas que logran desarrollar la capacidad de «liderazgo», entre estas podemos mencionar a Winston Churchill de Inglaterra o Vladimir Lenin de Rusia. Estos personajes demostraron su calidad como líderes de grandes masas y, por consiguiente, fueron base de ideas acerca del término «liderazgo» en tiempos posteriores. Aquella importante capacidad se refleja en nuestros tiempos en diversas situaciones, sean estas grandes o pequeñas. Hablamos de la manera de ejercer el «liderazgo» debido a la modernización de nuestra sociedad, esta ha implementado una demanda de líderes en diferentes entornos de nuestra vida diaria. Sin embargo, el concepto de liderazgo es uno muy amplio, sobre el cual muchos autores han demostrado, explicado, mencionado o ironizado. Pongo por caso a nuestro patrono Ricardo Palma en su obra Tradiciones peruanas, en la que el Bibliotecario Mendigo relata de forma breve nuestra etapa 
colonial. En las tradiciones menciona algunas anécdotas de los virreyes que estuvieron al mando en su momento, quienes ejercían un liderazgo distinto al de sus predecesores y, sin embargo, tenían influencia durante su mandato.

En este caso, se analizarán los relatos del decimoséptimo virrey del Perú, Luis Enríquez de Guzmán, en la tradición «Un virrey hereje y un campanero bellaco»; y la del decimoctavo virrey del Perú, Diego de Benavides y de la Cueva, en la tradición «La desolación de Castrovirreina», de tal manera que se pueda concluir cómo el liderazgo del virrey Luis Enríquez de Guzmán tuvo repercusión durante la toma de decisiones del virrey siguiente: Diego de Benavides y de la Cueva.

\section{SENTIDO DE LIDERAZGO}

Tal como se ha mencionado, el significado del término liderazgo no se puede establecer de manera concisa; sin embargo, podemos dar cuenta de este a partir de distintos puntos de vista de grandes autores. «El liderazgo no es más que una actividad o proceso de influenciar a la gente para que se empeñe voluntariamente en el logro de los objetivos del grupo» (González, 2014, p. 3). Para John Kotter el liderazgo se manifiesta a partir de la iniciativa de las personas para dejar de ser víctimas ante las circunstancias de la vida. Esto implica crear un ámbito en el cual los participantes logren desarrollar la capacidad de participar dentro de su entorno, manifestando así el término «otras realidades» con el fin de lograr una mejora dentro del entorno de trabajo continuo, con el objetivo de buscar nuevos métodos para realizar algún proceso en dicho entorno.

Así como Kotter define el liderazgo como una actividad, John Maxwell menciona, en su libro Las 21 leyes irrefutables del liderazgo, ciertas cualidades sobre el liderazgo: «Cuando un verdadero líder habla, la gente escucha, representa un aspecto de la ley de la influencia. 
La verdadera medida del liderazgo es influenciar» (2007, p. 19). Maxwell señala que el liderazgo más que una actividad dentro de un grupo, es una capacidad o habilidad que sirve como orientación para lograr un objetivo deseado. Además, resalta que el liderazgo representa una facultad humana con la finalidad de mejorar a las personas dentro de un sector a través de la orientación de un líder. Asimismo, define a un líder como aquella persona que logra la capacidad de influenciar a sus subordinados con la meta de que estos mejoren sus aptitudes y capacidades durante un periodo.

Dentro de los parámetros mencionados anteriormente, podemos afirmar que el liderazgo se define como aquella capacidad que logran algunas personas para poder orientar a grupos humanos con el fin de lograr ciertos objetivos; por otra parte, la finalidad del liderazgo es influenciar a dichas personas para que sigan ciertos ideales que llevarán a una mejora en las capacidades o habilidades de los seguidores. Sin embargo, existen diferentes métodos en los cuales se puede expresar la idea del liderazgo, algunos de estos suelen surgir a partir de situaciones espontáneas; por otro lado, existen otros que se dan mediante un proceso de aprendizaje experimental. A causa de la diversidad de modelos dados por el hombre durante su avance, se tomará dos modelos acerca del liderazgo con el fin de contrastarlos con las tradiciones «Un virrey hereje y un campanero bellaco» $\mathrm{y}$ «La desolación de Castrovirreina».

\section{LIDERAZGO TRANSFORMACIONAL}

Este modelo se origina por James MacGregor Burns en el año 1978 (Fischman, 2005, p. 18), quien lo define como aquel que se ejerce por personas que tienen una fuerte visión, así como una personalidad inquebrantable con la cual puedan cambiar las expectativas, percepciones o motivaciones de sus seguidores. También estableció los conceptos de liderazgo «transformacional» $\mathrm{y}$ «transaccional», en 
donde menciona que el modelo de transformación genera cambios significativos en las personas que lo ejerzan, en contraste con el transaccional, que se basa en el intercambio líder-subordinado con la finalidad de cumplir algún objetivo planteando, pero sin establecer una relación entre los individuos. También es considerado como un liderazgo "completo», esto se debe a que las características de estos líderes reflejan la idea de trabajar por un bien comunitario. En este modelo, la imagen del líder se ve plasmada como la de aquel que ejerce una figura inspiradora dentro de su entorno. Un ejemplo de este tipo de liderazgo lo podemos encontrar en la figura de Mahatma Gandhi: «Supongo que el liderazgo fue en un momento músculos destinados, pero hoy en día significa llevarse bien con la gente» (Frases go, s. f., párr. 11).

Ricardo Palma (2014) hace mención de este tipo de liderazgo en la tradición «La desolación de Castrovirreina», en la frase: «La ordenanza de obrajes, en protección de los infelices indios» (p. 349), que hace referencia al decimoctavo virrey Diego de Benavides y de la Cueva durante su mandato en el Perú. Como se puede advertir de los ejemplos dados, en ambos casos la imagen del liderazgo se ve enfocada en una mejora grupal, con el fin de desarrollar las capacidades de sus subordinados y que estos puedan lograr el objetivo que se han planteado.

\section{LIDERAZGO AUTORITARIO}

Este modelo se caracteriza por la autoridad que mantiene el líder, las ordenanzas de aquel son indiscutibles ante los demás por el hecho de ser el líder. Este tipo de liderazgo a lo largo de la historia ha sido catalogado como uno negativo, debido a que junto con él se desarrollaba el egocentrismo del líder. Un ejemplo de este liderazgo se da en Francia durante los años 1793 y 1794, en el denominado «Reinado del Terror», en donde Maximilien Robespierre gobernó 
de manera autoritaria. Dicho método trajo grandes consecuencias a Francia, debido a las muertes con la pena de la guillotina.

En la tradición «Un virrey hereje y un campanero bellaco» el autoritarismo se menciona de manera indirecta y de forma breve en la descripción del decimoséptimo virrey Luis Enríquez de Guzmán: «Los seis años de su administración fueron seis años de lágrimas, luto y zozobra pública» (Palma, 2014, p. 340). Es cierto que en este ejemplo la imagen del líder autoritario no se ve al mismo nivel que la de Robespierre, y esto se debe a que la imagen del virrey Luis Enríquez de Guzmán solo se relaciona con la falta de respeto que se le da a la religión dentro de la crónica, haciendo que su palabra sea mayor ante las creencias religiosas de su entorno.

Tal como se ha planteado, existen grandes diferencias entre estos dos tipos. Por un lado tenemos al liderazgo transformacional, el cual prioriza el bienestar del grupo de trabajo con finalidades próximas de logro, generando así una relación entre líder y subordinado para concluir en una mejora para ambos lados. Podemos resaltar la importancia que se ha dado a este modelo en tiempos modernos, debido a que en la actualidad se busca generar una conciencia grupal, de tal forma que todos logren generar capacidades para una mejora continua de un sector. Sin embargo, así como el liderazgo transformacional se enfoca en una mejora grupal, el modelo autoritario se concentra en una mejora personal dada por el líder. Este enfoque genera consecuencias positivas al igual que negativas, dentro de las cuales se tienen que tomar en cuenta algunas características, poniendo como ejemplo un líder autoritario que sabe de su campo y puede tomar decisiones buenas al igual que malas para un futuro, y esto se debe a su experiencia; sin embargo, si el líder no tiene experiencia, solo aumentará su egocentrismo, pero podría repercutir negativamente en su entorno ya sea a corto o largo plazo. 


\section{EL DESACUERDO SOCIAL CON UN LÍDER}

En la tradición «Un virrey hereje y un campanero bellaco» se mencionan las siguientes palabras:

El virrey conde de Alba de Liste no fue querido en Lima, por la despreocupación de sus ideas religiosas, creyendo el pueblo, en su candoroso fanatismo, que era él quien atraía sobre el Perú las iras del cielo. Y aunque contribuyó a que la Universidad de Lima, bajo el rectorado del ilustre Ramón Pinelo, celebrase con gran pompa el Breve de Alejandro VII sobre la Purísima Concepción de María, no por eso le retiraron el apodo de virrey hereje que un egregio jesuita, el padre Alloza, había contribuido a generalizar; pues habiendo asistido su excelencia a una fiesta en la iglesia de San Pedro, aquel predicador lo sermoneó de lo lindo porque no atendía a la palabra divina, distraído en conversación con uno de los oidores (Palma, 2014, p. 341).

Como se puede observar, el tradicionista resalta un conflicto idealista que existía por parte del virrey Luis Enríquez de Guzmán con la sociedad peruana de la época. Sin embargo, ¿por qué le da tanta importancia? Esto se debe a que los problemas mencionados en la tradición generan posteriormente una rivalidad entre el virrey y el pueblo. Tal es así que en la tercera parte de la tradición, Jorge Escoiquiz menciona:

Lo que soy yo, callaré como un difunto, que no me gusta informar a nadie de vidas ajenas; pero en lo que atañe al decoro de mis campanas, no cedo ni el canto de una uña, que nos las fundió el herrero para rufianas y tapadoras de paseos pecaminosos. Si vuecencia no quiere que ellas den voces, facilillo es el remedio. Con no pasar por la plazuela salimos de compromisos (Palma, 2014, p. 344).

Generando de tal forma un trato conveniente para «el campanero bellaco» por las deficiencias sociales que existían entre el virrey y el pueblo. Por el contrario, en la tradición «La desolación de 
Castrovirreina» se muestra una relación entre el virrey y el pueblo en la frase: «La ordenanza de obrajes, en protección de los infelices indios, y la habilidad con que administró las rentas públicas, llegando a tener el tesoro en vez de déficit un sobrante de medio millón, basta para hacer la apología de este virrey» (Palma, 2014, p. 349). La relación entre el virrey y el pueblo se ve reflejada en lo que se logró conseguir a causa del apoyo social.

\section{EL LIDERAZGO VISTO SEGÚN LAS TRADICIONES PERUANAS}

Analizando lo propuesto, la imagen del liderazgo se ve reflejada en las Tradiciones peruanas de Ricardo Palma (2014) en la crónica del decimoséptimo virrey del Perú Luis Enríquez de Guzmán, en la que el tradicionista menciona ciertas características importantes del virrey: «Magistrado de buenas dotes administrativas y hombre de ideas algo avanzadas para su época» (p. 340), que se refiere a las grandes cualidades como líder; por el contrario, su imagen de líder se veía afectada debido a la falta de interés del virrey por la religión, lo cual dio lugar al sobrenombre de «virrey hereje». Sin duda, Luis Enríquez de Guzmán tenía las cualidades necesarias para destacar como un buen líder, pero el desapego a la religión influyó en su mandato, dando lugar a un aumento en el egocentrismo del virrey, gracias a que era el primer grande de España que venía al Perú. Cabe resaltar que durante su periodo como gobernante, sucedieron grandes problemas sociales con respecto al virrey.

Cuando Diego de Benavides y de la Cueva entra al poder, este se ve influenciado por los problemas de su predecesor, generando así una imagen de un líder transformacional para poder así solucionar dichos problemas y crear un ambiente de armonía durante su mandato. Estas características se ven reflejadas en la descripción que se le da al virrey, lo que genera una clara preocupación sobre sus subordinados. 


\section{IMPORTANCIA DEL LIDERAZGO}

Sin lugar a dudas podemos resaltar la importancia que ha tenido el líder a lo largo de la historia: cada rol de este ha ido variando según las épocas, de tal manera que algunas han sido complemento de nuevas teorías del liderazgo y otras han servido como base para una nueva teoría. En la actualidad se busca que el grupo sea aquel que se apoye, con la finalidad de lograr una mejora en las capacidades de los involucrados, y no solo del líder, como se menciona en el modelo autoritario de liderazgo. Convenientemente, el modelo transformacional ha sido descrito antes de la definición hecha por MacGregor, lo cual se ve reflejado en la imagen del virrey Diego de Benavides y de la Cueva, lo que sería en la actualidad un modelo de liderazgo muy demandado.

A pesar de eso, un líder ve influenciada su toma de decisiones por el liderazgo empleado por sus antecesores, de tal manera que las decisiones tomadas influyen de cierta forma en futuras acciones, generando nuevos ideales a consecuencia de estos. De igual modo, a lo largo de nuestra historia, las personas han realizado dicho proceso indirectamente. Así, un ideal logra una cima del conocimiento que luego pasa a ser la base de una nueva ideología. Un gran ejemplo de este proceso se ha dado con respecto a la forma de liderar. Los griegos mantenían una forma de liderar políticamente correcta, basada en el orden de la república, donde el líder tenía que ser justo con respecto a los demás, hasta aquellos líderes que buscaban el poder absoluto para alimentar su egocentrismo, tal es el caso del zar Nikolái Aleksándrovich Románov, que ante el peligro de una revolución tuvo que tomar acciones contra los manifestantes para poder mantener su poder autoritario en Rusia.

Volviendo al tema central de este artículo, el tradicionista hace referencia a un contraste idealista: por un lado, tenemos la crónica del decimoséptimo virrey Luis Enríquez de Guzmán en la tradición 
«Un virrey hereje y un campanero bellaco», que representa el modelo autoritario de liderazgo por las menciones que se le atribuyen en la crónica. Por el otro lado, tenemos la crónica del decimoctavo virrey Diego de Benavides y de la Cueva en la tradición «La desolación de Castrovirreina», en la que se hace referencia a las ideas del modelo transformacional de liderazgo, en las preocupaciones que existían por parte del virrey hacia sus subordinados. En ambos casos Palma hace uso de diversas herramientas literarias para expresar de manera indirecta la importancia del virrey.

Para terminar, resalto la importancia que ha tenido el líder desde los comienzos de la humanidad. Si no fuera por el impulso de los líderes, muchas decisiones no se hubieran tomado a lo largo de la historia, gracias a estos el ser humano ha sido beneficiado en diversos entornos; sin embargo, este ideal de «liderazgo» también ha traído algunas contradicciones en épocas anteriores. Es así como el liderazgo ha pasado por diversos cambios con diferentes enfoques. Hoy en día destacan diversos tipos de líderes mundiales, un ejemplo de estos líderes son Bill y Melinda Gates. De estos resalta su enfoque en torno al sector social. La revista Fortune menciona lo siguiente:

Su trabajo durante los últimos 20 años ha ayudado a transformar las vidas de cientos de millones de personas, y con certeza afectarán a miles de millones más si la investigación que ellos financian en la actualidad ayuda a prevenir y curar el sida, la tuberculosis y la gripe. Ayudarán inmensamente más si el trabajo que hacen para que las mujeres cobren poder, para brindar higiene, para impulsar la agricultura y para mejorar la educación (y el acceso a la educación) dan frutos plenos (2019, p. 44).

Reafirmando que como líderes tienen una visión de mejora continua que impulsa a gente que necesita esa orientación. Esto se debe a la motivación que emiten a través de sus acciones, aquellas acciones que repercutirán en un futuro para mejorar la sociedad. 


\section{REFERENCIAS}

Chiavenato, I. (2002). Administración: teoría, proceso y práctica. Traducción de Germán Alberto Villamizar. McGraw-Hill.

Fischman, D. (2005). El líder transformador II. Planeta.

Fortune en español (2019). World's 50 greatest leaders. Fortune en Español, 40-44.

Frases go (s. f.). Frases de Mahatma Gandhi. https://www.frasesgo. com/autores/frases-de-mahatma_gandhi-5.html

González, Y. (2014). ¿Cómo inciden los diferentes tipos de liderazgo en el comportamiento profesional y personal de los empleados en las entidades del sector cooperativo que existen en Colombia? https:// repository.unimilitar.edu.co/bitstream/handle/10654/13210/ ENSAYO\%20LIDERAZGO.docx.pdf? sequence $=1$

López, E. (2013). La importancia del liderazgo en las organizaciones. Universidad Autónoma de Barcelona. https://ddd.uab.cat/pub/ tfg/2013/110463/TFG_elopezmartinez.pdf

Maxwell, J. (2007). Las 21 leyes irrefutables del liderazgo. Grupo Nelson.

Maxwell, J. (2016). Liderazgo 101: lo que todo líder necesita saber. Grupo Nelson.

Mercedes, M. (2018). Lo que llamamos un líder: consideraciones críticas de lo que ocurre en diversas organizaciones. Perspectivas Psicológicas, 3-4, 134-144. http://pepsic.bvsalud.org/pdf/pp/v3-4/ v3-4a15.pdf

Palma, R. (2014). Un virrey hereje y un campanero bellaco y La desolación de Castrovirreina. En Tradiciones peruanas. Primera y segunda series (M. Á. Rodríguez Rea, ed.; pp. 337-345, 347-352). Editorial Universitaria de la Universidad Ricardo Palma. 